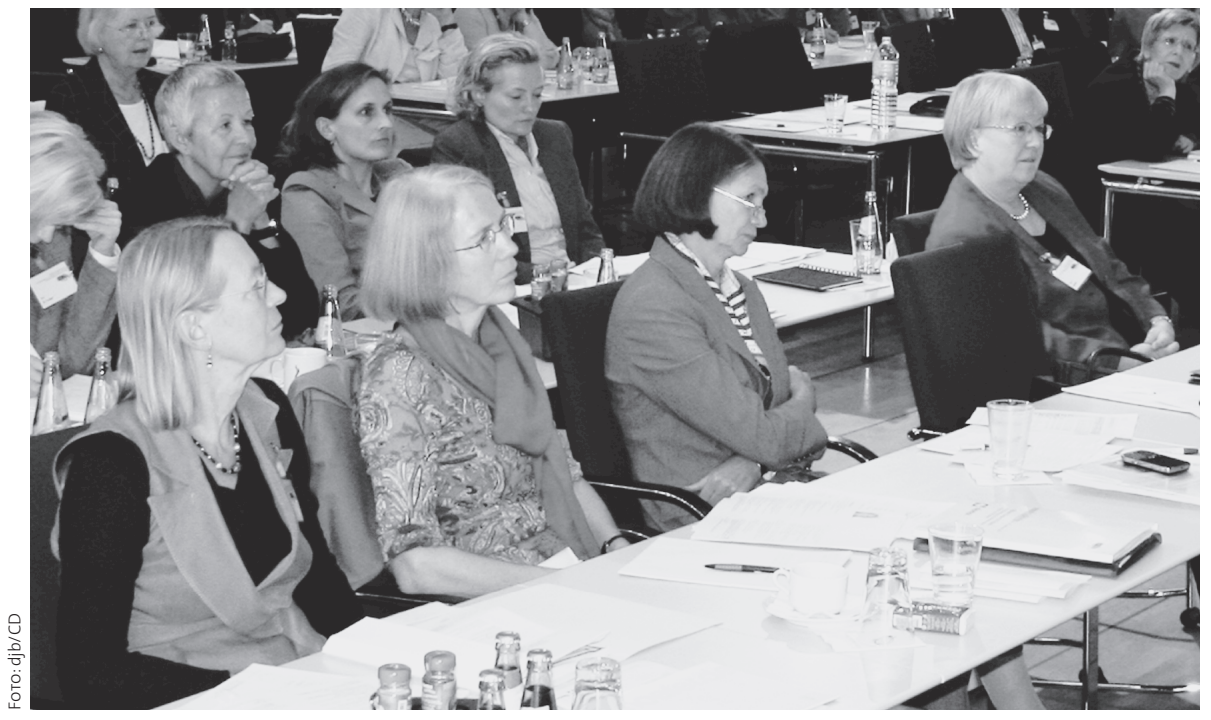

4 Die Tagung am 23. September 2011 im Kongresshotel Potsdam: vorne im Bild Prof. Dr. Sibylle Raasch (Universität Hamburg), Dr. Regine Winter (Richterin am Bundesarbeitsgericht), Jutta Wagner, Renate Augstein (Referatsleiterin im BMFSFJ) (v.I.n.r.)

politisch verfehlt sind. Unternehmen und Verwaltungen sollen sich in ihren unterschiedlichen Ausgangsbedingungen ernst genommen und ihre Vielfalt Berücksichtigung finden. Die selbst gesetzten Ziele müssen veröffentlicht und in Berichten evaluiert werden. Diese Veröffentlichungspflicht sichert die notwendige Transparenz des Verfahrens und schafft Wettbewerb bei den Akteurinnen und „Die Ziele des Bundesgleichstellungsgesetzes und des Bundesgremienbesetzungsgesetzes werden mit Nachdruck verfolgt. Wir werden prüfen, ob und inwieweit die Gesetze geändert und effektiver gestaltet werden müssen. Der Anteil von Frauen in Führungspositionen in der Wirtschaft und im öffentlichen Dienst soll maßgeblich erhöht werden. Dazu wird ein Stufenplan, insbesondere zur Erhöhung des Anteils von Frauen in Vorständen und Aufsichtsräten vorgelegt.“

Im Rahmen dieses Stufenplans beabsichtigt das Bundesfrauenministerium, noch im Jahr 2011 eine gesetzliche Regelung, synchron für Privatwirtschaft und öffentliche Hand, zur Erhöhung des Frauenanteils in Führungspositionen vorzulegen. Der Gesetzentwurf wird Verpflichtungen für Unternehmen und Bundesverwaltung vorschreiben, sich selbst konkrete Ziele zur Erhöhung des Frauenanteils in Führungspositionen zu setzen. Dem liegt der Ansatz zugrunde, dass starre Einheitsquoten für die Gesamtheit der Unternehmen und Verwaltungen ordnungs-

Akteuren. Angesichts des bevorstehenden Fachkräftemangels werden es sich weder private noch öffentliche Arbeitgeber in Zukunft leisten können, gut qualifizierte Frauen durch „gläserne Decken“ zu verlieren. Bei einer Nichteinhaltung der selbst gesetzten Ziele werden Sanktionen greifen, denn wir wissen aus verschiedenen Studien und Erfahrungen anderer Länder, dass reine Quotenregelungen ohne Sanktionen kaum Wirkung entfalten.

Verehrte Mitglieder des djb, wir wollen - auch mit Ihrer Fachkompetenz und Ihrem Engagement - die Chance jetzt nutzen und ein Gesetz zur Erhöhung des Frauenanteils in Führungspositionen auf den Weg bringen, damit Gleichstellung in den Entscheidungszentren unserer Gesellschaft schneller und nachhaltig erreicht wird.

In diesem Sinne wünsche ich uns allen guten Mut, viel Kreativität und Durchsetzungskraft bei der Verfolgung unserer gemeinsamen Ziele - und natürlich eine spannende Fachtagung!

\title{
Foren Zugang und Aufstieg im öffentlichen Dienst und in anderen Bereichen
}

\section{Dr. Katja Rodi}

Vorsitzende der Kommission Öffentliches Recht, Europa- und Völkerrecht; Wissenschaftliche Mitarbeiterin, Universität Greifswald

\section{Maren Thomsen}

Mitglied der Kommission Öffentliches Recht, Europa- und Völkerrecht; Richterin am Bundesverwaltungsgericht, Richterin am Landesverfassungsgericht Schleswig-Holstein, Leipzig/Kiel

\section{Die Zahlen - keine gleichen Karrierechancen für Frauen im öffentlichen Dienst}

Ziel der Fachtagung war es unter anderem, die Mechanismen und Ergebnisse zu beleuchten, denen sich Frauen beim Zugang und beim Aufstieg im öffentlichen Dienst zu stellen haben, kurzum: zu beleuchten, wieso Frauen nicht die gleichen Karrierechancen im öffentlichen Dienst haben wie Männer. Zugleich sollte auf andere Bereiche geblickt werden, um zu schauen, ob sich hier für Juristinnen Alternativen auftun, aber auch, ob es Übertragungsmöglichkeiten aus diesen Bereichen auf den öffentlichen Dienst im allgemeinen oder die einzelne Frau im öffentlichen Dienst und umgekehrt gibt.

Denn die Zahlen im öffentlichen Dienst sind eindeutig und die sich daraus ergebenden Befunde erschreckend. Sie zeigen, dass es Gründe geben muss, die Frauenkarrieren verhindern und gleichzeitig Männerkarrieren massiv erleichtern. Erst wenn diese Gründe bekannt sind, kann über Abhilfemaßnahmen bis hin zu Gesetzesinitiativen nachgedacht werden. Die 
Tagung zeigte, dass hier noch Vieles im Unbekannten liegt. Gelegentlich entsteht der Eindruck, dass die Benachteiligung von Frauen bewusst verschleiert und unter vielem Zahlenund Papiermaterial und einem riesigen Gleichstellungsapparat vergraben wird, mit dem die Frauen beruhigt werden sollen. Der Vortrag von Prof. Dr. Silke Ruth Laskowski ${ }^{1}$ belegte sehr eindrucksvoll, dass sowohl das Bundesgleichstellungsgesetz mit der dort installierten Gleichstellungsbeauftragten als auch das Bundesgremienbesetzungsgesetz ${ }^{2}$ zahnlose Tiger sind. Die von Prof. Dr. Laskowski aufgezeigten Lösungsmöglichkeiten sind vom djb einzufordern.

Die Fakten, die die massive Benachteiligung von Frauen im öffentlichen Dienst belegen, müssten skandalisiert und eine jährliche Peinlichkeitsrangliste für den öffentlichen Dienst aufgestellt werden. Die Wählerinnen müssten im Grunde die zuständigen Politiker/innen für ihr Verhalten abstrafen, denn jede staatliche Gewalt bedarf demokratischer Legitimation. Die öffentliche Verwaltung einschließlich der dritten Gewalt - der öffentliche Dienst - handelt/n im Namen und im Auftrag des deutschen Volkes.

Art. 20 GG lautet:

(1) ...

(2) Alle Staatsgewalt geht vom Volke aus. Sie wird vom Volke in Wahlen und Abstimmungen und durch besondere Organe der Gesetzgebung, der vollziehenden Gewalt und der Rechtsprechung ausgeübt.

(3) Die Gesetzgebung ist an die verfassungsmäßige Ordnung, die vollziehende Gewalt und die Rechtsprechung sind an Gesetz und Recht gebunden.

Die Beamtinnen und Beamten, die Richterinnen und Richter werden deshalb vom Bundespräsidenten, von den Ministerpräsidentinnen und Ministerpräsidenten der Länder etc. im Namen der zuständigen Gebietskörperschaft (Bundesrepublik Deutschland, Bundesland, etc.) ernannt und befördert. Die Verwaltung ist an Recht und Gesetz gebunden, und hierzu gehört auch Artikel 3 des Grundgesetzes, der lautet:

(1) Alle Menschen sind vor dem Gesetz gleich.

(2) Männer und Frauen sind gleichberechtigt. Der Staat fördert die tatsächliche Durchsetzung der Gleichberechtigung von Frauen und Männern und wirkt auf die Beseitigung bestehender Nachteile hin.

(3) Niemand darf wegen seines Geschlechtes, seiner Abstammung, seiner Rasse, seiner Sprache, seiner Heimat und Herkunft, seines Glaubens, seiner religiösen oder politischen Anschauungen benachteiligt oder bevorzugt werden. Niemand darf wegen seiner Behinderung benachteiligt werden.

Die Zahlen belegen, der öffentliche Dienst, zugleich einer der größten Arbeitgeber, wird trotz dieser eindeutigen Verfassungsvorgaben seiner Vorbildfunktion nicht gerecht. Im europäischen Vergleich befindet er sich in der drittletzten Position; nur Belgien und Luxemburg hatten schlechtere Ergebnisse. ${ }^{3}$ Eigentlich müsste deshalb ein Aufschrei durch die Republik gehen. Ministerinnen und Minister müssten reihenweise zurücktreten. Aber: Bisher scheint diesem Thema zu wenig Aufmerksamkeit entgegengebracht zu werden, die Berichte, Zah- len und Gutachten verschwinden ungelesen in Papierkörben, Gleichstellungsbeauftragte werden nicht gehört, nichts geschieht ...

Besonders unglaubwürdig wird es, wenn der öffentliche Dienst sich dann auch noch als Vorbild für die Privatunternehmen geriert und dort mehr Frauen in Führungspositionen fordert. (Nur wenn der Begriff „Führungsposition“ weiter gefasst wird, steht der öffentliche Dienst im Vergleich zu den Privatunternehmen etwas besser da.) Weder bei der eigenen Personalverwaltung, noch bei der Gremienbesetzung, noch in den privatwirtschaftlich tätigen Unternehmen in öffentlicher Hand, noch bei der öffentlichen Auftragsvergabe erfüllt der öffentliche Dienst selbst die Vorgaben des Artikels 3 Absatz 2 GG, sondern scheint ein Karriereclub für Männer zu bleiben, der gelegentlich eine, manchmal auch ein wenig mehr Frauen Karriere machen lässt, um den Rest der Frauen und das eigene Gewissen zu beruhigen.

Dies muss sich ändern!

\section{Zugang}

Im Forum „Zugang“ waren vertreten: Die klassische Verwaltung durch Elke Gündner-Ede, Mitglied des geschäftsführenden Bundesvorstands der Gewerkschaft der Polizei, die Wissenschaft durch Dr. Ulrike Eickhoff, Leiterin der Stabsgruppe Qualitätssicherung und Verfahrensentwicklung bei der Deutschen Forschungsgemeinschaft, der freie Beruf der Rechtsanwältin durch Rechtsanwältin Margret Diwell, Präsidentin des Verfassungsgerichtshofs Berlin, und die privaten Unternehmen durch Ursula Matthiessen-Kreuder, Leiterin Personal der CSC Deutschland in Wiesbaden.

Bei den beiden im ersten Forum nicht vertretenen klassischen Justizberufen (Richterin und Staatsanwältin) erzielen Frauen mittlerweile beim Zugang vorbildliche Ergebnisse: teilweise über 50 Prozent Frauen als Proberichterinnen, das Ganze ohne Quoten und ganz unabhängig von den unterschiedlichen Einstellungsverfahren, allein wegen der besseren Examensnoten der Frauen.

Insgesamt ist aufgrund der Vorgabe des Artikels 33 Absatz 2 GG, der lautet

„Jeder Deutsche hat nach seiner Eignung, Befähigung und fachlichen Leistung gleichen Zugang zu jedem öffentlichen Amte“ der Zugang zum - wie der Aufstieg im - öffentlichen Dienst stark abhängig von Beurteilungen. ${ }^{4}$ Nur bei gleicher Beurteilungslage, also wenn die Bewerberinnen und Bewerber im Wesentlichen gleich beurteilt sind, kommen Quotenregelung im öffentlichen Dienst überhaupt in Betracht. Selbst dann lassen sich im Zweifel genügend Gründe finden, um eine

1 Gleichstellungsrechtliche Instrumentarien im öffentlichen Dienst Überblick und Bewertung der Steuerungsqualität. Abdruck in der nächsten djbz.

2 Vgl. hierzu auch den 2. Erfahrungsbericht zum Bundesgleichstellungsgesetz, BT-Drs. 17/4307, und den 5. Gremienbericht, BT-Drs. 17/4308, beide vom 16. Dezember 2010.

3 Vgl. BT-Drs. 17/4307, S. 21.

4 Vgl. BVerfGE 110, $304\langle 332\rangle$, st. Rspr. vgl. auch BVerwG, zuletzt Urteil v. 30.6.2011 - BVerwG 2 C 19.10 - Rn. 15 und 16. 
Quote nicht anzuwenden, wie auch die bisherige Rechtsprechung verdeutlicht.

Da die Beurteilungen der Frauen - und dies nicht nur im Justizbereich - am Anfang der Berufskarrieren mittlerweile aufgrund einer stark verschulten Ausbildung und teilweise anonymisierter Prüfungsverfahren (Hoch- und Fachhochschulabschlüsse, staatliche Examina, Diplomprüfungen etc.) meistens besser als diejenigen der Männer sind, haben die Frauen in der Regel auch die besseren Chancen bei einer Einstellung. Dies bleibt auch so, wenn die Dienstherren zusätzliche Hilfskriterien (wie Bewerbungsgespräche, AssessmentCenter, Prüfungsarbeiten o.ä.) heranziehen, weil sich die Frauen hierauf hochprofessionell vorbereiten und deshalb auch dort oft besser, zumindest aber gleich gut abschneiden.

Dies alles ist eine sich schon seit mindestens 15 Jahren abzeichnende durchgehende Tendenz. Sie wurde bei den Juristinnen damit eingeleitet, dass mehr Frauen in das Jurastudium gingen und in die Noten für das Zweite Staatsexamen nicht mehr (früher sogar hauptsächlich) die Stationsnoten einflossen, sondern neben der mündlichen Prüfung allein und maßgeblich anonyme Prüfungsarbeiten zählten. Daraus die Folgerung zu ziehen, dass nur noch anonyme Prüfungsarbeiten entscheidend sein dürften, verkennt, dass diese nur einen von der Tagesform abhängigen Ausschnitt der beurteilten Person abbilden können, die möglicherweise auch noch durch Ängste vor schriftlichen Arbeiten verfälscht werden können. Aber: Berufszugänge, die zu einem hohen Anteil von anonymisierten Prüfungsverfahren bestimmt werden, zeichnen sich durch gleichmäßige Erfolgsquoten für beide Geschlechter aus und entsprechen damit tendenziell eher und sicherer den verfassungsrechtlichen Vorgaben des Artikels 3 Absatz 2 GG.

Elke Gündner-Ede stellte die Zugangsvoraussetzungen im Polizeidienst dar und bestätigte, dass hierbei wie auch beim Zugang zum mittleren (soweit noch vorhanden) als auch beim gehobenen Dienst kaum Benachteiligungen von Frauen zu verzeichnen sind. Schwerer gestaltet sich das bei der Bewerbung zum höheren Dienst, da für die Frauen neben den formalen Voraussetzungen wie Beurteilungen und Potenzanalysen auch Erschwernisse in ihrer individuellen Lebensplanung liegen. So bedeutet im Polizeibereich die Wahl einer höheren Laufbahn am Anfang eine stark verschulte Ausbildung an einem zentralen Ort, was Frauen im Hinblick auf ihre Familienplanung und -pflichten oft ablehnen müssen. Hier muss Abhilfe durch veränderte Rahmenbedingungen eines Masterstudiums - wie zum Beispiel Fernstudium als eine Möglichkeit -, aber auch eine Änderung der gesamtgesellschaftlichen Verhältnisse im Hinblick auf die Zuständigkeiten in der Familie geschaffen werden. Wie in anderen europäischen Ländern müssen beide Geschlechter sich für die Kindererziehung gleichermaßen zuständig fühlen und es muss mehr und bessere Angebote für die Kinderbetreuung geben.

Ein Hauptproblem bei der Einstellung oder dem Aufstieg ist für Frauen aus der forensischen Praxis dann auch eher das Altersproblem durch familienbedingte Ausfallzeiten, wenn diese Zeiten nicht angerechnet werden. Denn generell gibt es (unterschiedliche) Höchstaltersgrenzen beim Zugang für die einzelnen Beamten-/Richterlaufbahnen, die die Frauen durch diese (Ausfall-)Zeiten überschreiten können.

Ulrike Eickhoff beleuchtete den für den Zugang zum öffentlichen Dienst untypischen Wissenschaftsbereich. Die Ernennung zur Professorin stellt nicht den Anfang, sondern den Höhepunkt der wissenschaftlichen Karriere dar. Eickhoff zeigte ein besonders machtvolles Instrument auf, mit dem sich binnen kurzer Zeit bereits deutlich messbare Veränderungen im Geschlechterverhältnis erreichen ließen: Geld! Aufgrund einer Selbstverpflichtung der Wissenschaft ist die Vergabe von Fördermitteln durch die Deutsche Forschungsgemeinschaft, einer sehr bedeutenden Geldgeberin in diesem Bereich, an die Einhaltung „Forschungsorientierter Gleichstellungsstandards“ gebunden. Die unzureichende Beteiligung von Frauen in der Wissenschaft wird als Verlust hervorragender Talente verstanden, als Effizienz- und Exzellenzdefizit.

Diese Idee lässt sich vielfältig übertragen: So könnte Geld als Anreiz übertragen werden auf die Vergabe öffentlicher Mittel. Frauenförderung als Kennzeichen exzellenter Forschung könnte übertragen werden auf die Förderung von Führungskräften: Nur dann, wenn diese Frauen ausreichend fördern, können sie selbst weiter befördert werden (wobei jede Beförderung neben Prestige auch wiederum ein finanziellen Vorteil bedeutet). In Kombination mit dem von Elke GündnerEde aus dem niedersächsischen Polizeidienst vorgestellten Mentoringprogramm ließen sich hier binnen kurzer Zeit die Zahlen der Beförderungen von Frauen in den Normalbereich anheben. Und eine jährliche Rankingliste, wie sie die Deutsche Forschungsgemeinschaft für die Verwirklichung der Gleichstellungsstandards an den Universitäten jährlich veröffentlicht, ist auch im öffentlichen Dienst möglich und bliebe sicher nicht wirkungslos.

Margret Diwell und Ursula Matthiessen-Kreuder zeigten auf, welche Bereiche neben dem öffentlichen Dienst für Juristinnen hervorragende alternative Tätigkeitsfelder mit guten Karrierechancen anbieten. Der Zugang zu diesen Berufen (Rechtsanwältin, privatrechtlich organisierte Unternehmen) fordert von der Einzelnen Mut und ausreichende Information über den künftigen Beruf. So ist die Zahl von Partnerinnen in größeren Anwaltskanzleien erschreckend niedrig, so dass sich im Anwaltsberuf vor der Selbständigkeit der Einstieg als Angestellte anbietet. In privatrechtlichen Unternehmen sollten nicht diejenigen Sparten angesteuert werden, in denen Frauen überproportional vertreten sind, weil dies auf Dauer einen ökonomischen Nachteil und zudem weniger Karrierechancen bedeutet; dies lässt sich für die einzelne Frau unproblematisch auch auf den öffentlichen Dienst übertragen.

\section{Aufstieg/Karrieren}

Im Forum Aufstieg befassten sich die Bundestagsabgeordnete und vormalige Bundesjustizministerin Brigitte Zypries sowie die Vorsitzende Richterin am Oberlandesgericht und neue Präsidentin des djb Ramona Pisal mit der Bundesverwaltung und der Richterschaft. Rechtsanwältin und Notarin und Mit- 
glied des Bundesvorstandes des Deutschen Anwaltvereins Mechtild Düsing zeigte die Situation von Frauen in den Berufen der Rechtsanwältin, der Notarin und der Wirtschaftsprüferin und Verbesserungsmöglichkeiten auf. Ursula Matthiessen-Kreuder berichtete über Karrieremöglichkeiten in Unternehmen in privater Rechtsform.

Auch beim Aufstieg im öffentlichen Dienst spielt Artikel 33 Absatz 2 GG, also die Bestenauslese, eine zentrale Rolle. Die dabei ausschlaggebenden Beurteilungen sind bundesweit und auch innerhalb der verschiedenen Bereiche im öffentlichen Dienst sehr unterschiedlich gestaltet, von einer einheitlichen Verbalbeurteilung bis hin zum Punktesystem mit Einzelmerkmalen.

Da schon zumindest seit den Neunzigerjahren im Bereich der Gerichte und Staatsanwaltschaften bei den Einstellungen der Anteil von Frauen und Männern - und zwar aufgrund der Bestenauslese - in etwa gleich hoch ist, müssten diese hoch qualifizierten Frauen in etwa in gleichem Umfang wie die mit ihnen neu eingestellten Männer Karriere gemacht haben. Hiervon ist der öffentliche Dienst aber weit entfernt. Ramona Pisal und Brigitte Zypries bestätigten für die Gerichte und die Bundesverwaltung eine zahlenmäßig immer stärker werdende Unterrepräsentanz von Frauen, je höher die Position in der jeweiligen Karriereleiter ist. ${ }^{5}$ Wenn auch die beiden Referentinnen - ebenso wie später im Verlauf der Tagung die Abteilungsleiterin im Bundesministerium für Familie, Senioren, Frauen und Jugend Eva Maria Welskop-Deffaa (Ergebnisse und Einschätzungen in den Berichten zum BGleiG und BGremG) - eine geringe, aber gleichwohl zahlenmäßig bemerkbare Steigerung des Frauenanteils in Führungspositionen darstellen konnten, bleiben die Zahlen weiterhin erschreckend niedrig. So ist Eva Maria Welskop-Deffaa die einzige Abteilungsleiterin in ihrem Ministerium (neben fünf Männern) und bei der letzten Bundesrichterwahl waren unter den 18 neu gewählten Richterinnen und Richtern nur drei Frauen. Als Ursache hierfür benannte Brigitte Zypries, dass selbst bei einem entsprechenden politischen Willen oftmals nicht genug oder sogar keine in Frage kommenden Frauen zur Verfügung standen. So sind unter den Wahlvorschlägen der Länder - wenn auch von Land zu Land unterschiedlich - zur Bundesrichterwahl anteilsmäßig insgesamt nur sehr wenig Frauen vertreten. ${ }^{6}$ Brigitte Zypries bat den djb um Benennung geeigneter Kandidatinnen, ein Projekt, dessen sich Bundesanwältin (GBA) und neue Vizepräsidentin des djb Eva Schübel angenommen hat.

Allgemein sind Quotenregelungen im öffentlichen Dienst vor den Gerichten auch beim Aufstieg wenig erfolgreich, obwohl es immer wieder versucht wird. So war eine Regelung der Bundeszollverwaltung Gegenstand gerichtlicher Entscheidungen, nach der bei gleicher aktueller und Vorbeurteilung erst die behinderten Frauen, dann die Frauen, dann die behinderten Männer, dann die restlichen Männer befördert werden sollten und innerhalb der so gebildeten Untergruppen dann weiter nach Dienst- und Lebensalter differenziert wurde. ${ }^{7}$ Das Problem im Hinblick auf die Quote war hier, dass aufgrund eines zu grob gewählten Rasters ungefähr 300 Bewerberinnen

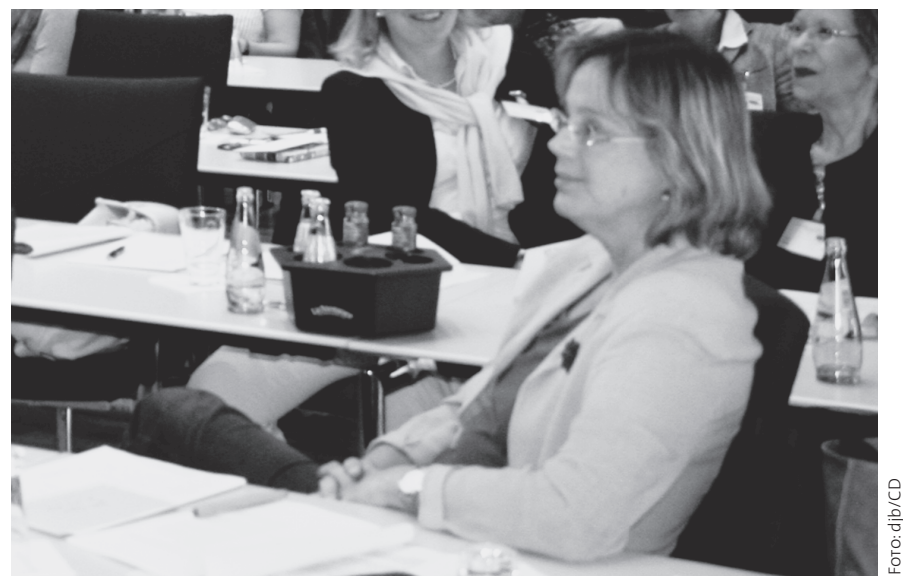

- Vorne im Bild: Eva M. Welskop-Deffaa (Abteilungsleiterin Gleichstellung, Chancengleichheit, Bundesministerium für Familie, Senioren, Frauen und Jugend, Berlin)

und Bewerber gleich beurteilt waren, damit also entweder die Beurteilungen nicht hinreichend differenzierten oder aber die Beurteilungen nicht hinreichend ausgewertet worden waren, die Quote also nicht angewendet werden durfte. Denn diese kann erst eingreifen, wenn die Bewerber/innen im Wesentlichen gleich sind.

Außerdem verursachen beamtete Frauen im gebärfähigen Alter bei Personalplanern im öffentlichen Dienst erhebliches Kopfzerbrechen. Diese reduzieren oft ihre Arbeitszeit. Es gibt jedoch - und zwar durch das Grundgesetz geschützt ${ }^{8}$ - einen Anspruch teilzeitbeschäftigter Beamtinnen und Beamter auf Rückkehr zur Vollzeit, und zwar jederzeit, spätestens zum nächsten Haushaltsjahr. ${ }^{9}$ Diese Vorschrift macht zugleich bei Beamtinnen und Richterinnen eine gezielte Ausschreibung von Beförderungsstellen als Halbtagsstellen schwierig.

Obwohl die Frauen am Anfang ihres Berufslebens im Vergleich zu den Männern mit besseren Beurteilungen starten, kehrt sich das Verhältnis im Laufe ihres Berufslebens um. Familienbedingte Unterbrechungen oder Reduzierungen sind immer noch häufiger bei Frauen zu finden und führen manchmal zu Altersproblemen beim Laufbahnwechsel. Sie führen aber auch zu Ausfallzeiten in den Beurteilungen. Da Beurteilte in der Regel von Beurteilung zu Beurteilung besser werden, könnten und müssten diese Benachteiligungen in begrenztem Umfang durch fiktive Fortschreibungen der Beurteilungen aufgehoben werden. ${ }^{10}$ Frauen sind in den Beurteilungen in den beförderungsrelevanten Spitzennoten nicht entsprechend ihrem Anteil in den jeweiligen gleichen Statusämtern vertre-

\footnotetext{
5 Aktuelle Zahlen mit Entwicklungstendenzen für den Bereich der Bundesverwaltung: vgl. insbes. BT-Drs. 17/4307, für die Gerichte und Staatsanwaltschaften auf der Homepage des Bundesjustizministeriums: <http://www.bmj.de/DE/Service/StatistikenFachinfaormationenPublikationen/Statistiken/Personal/_node.html> (Zugriff: 10.10.2011); vgl. auch zu den Gerichten BT-Drs. 17/5874.

6 Es gibt Länderquoten, vgl. Art. 36 Abs. 1 S. 1 GG.

7 Vgl. BVerwG, Urteil v. 30.6.2011 - BVerwG 2 C $19.10-$

8 Art. 33 Abs. 5 GG.

9 StRspr., vgl. zuletzt BVerwG, Urteil v. 24.2.2011 - BVerwG 2 C - 50.09 - Rn. 20-24.

10 Zu den Grenzen: vgl. BVerwG, Urteil v. 16.12.2010 - BVerwG 2 C 11.09-.
} 
ten; Teilzeitbeschäftigte werden in der Regel schlechter beurteilt. ${ }^{11}$ Dies ist auf beiden Seiten (Beurteilte/r und Beurteiler/ in) vielfach nicht bekannt. Hier hilft eine regelmäßige Evaluation der Beurteilungen und ein Öffentlichmachen der Beurteilungsergebnisse, soweit dies mit dem Personaldatenschutz vereinbar ist. Beurteilungen sind durch vielfache Zielvorgaben vorgeprägt, so dass eine Einführung von Geschlechterquoten als weitere Zielvorgabe gefordert werden muss. ${ }^{12}$

Auch individuelle, familienbedingte Besonderheiten verhindern gute Beurteilungen und damit gute Karrierechancen. So wird Frauen nach der Rückkehr aus der Elternzeit oft die vorherige Stelle erneut angeboten, so dass sie keine Verwendungsvielfalt aufweisen können und in der Behörde eher unbekannt bleiben. Örtliche karrierefördernde Wechsel wie Erprobungen an Obergerichten sehen sie als organisatorisch schwer zu bewerkstelligen an, zumal diese außerdem nur selten in Teilzeit angeboten werden. Generell werden Ortswechsel zum Aufsteigen auf der Karriereleiter von Frauen häufiger abgelehnt als von Männern. Ramona Pisal wies auf Frauenförderpläne (mit Berichtspflichten), gezielte Personalführungsgespräche und Mentoring als Abhilfemöglichkeiten hin.

Hieran schlossen die Berichte von Ursula MatthiessenKreuder und Mechtild Düsing an. Matthiessen-Kreuder widmete sich der Frage, wie Frauen in Unternehmen in privater Rechtsform Karriere machen. Sie verdeutlichte sehr eindrucksvoll, dass am wenigsten die fachliche Eignung, also gutes und fleißiges Arbeiten, und auch eher nachrangig die Führungskompetenz ausschlaggebend für eine Karriere sind. Mentoring, Netzwerke, Flexibilität, Rekrutierung waren hier die Zauberworte, die Frauen individuell voranbringen. Ihre Beobachtungen lassen sich wiederum vielfach auf den öffentlichen Dienst übertragen und helfen Frauen auch dort, schon jetzt ihre individuellen Karrierechancen zu verbessern. Mechtild Düsing zeigte, dass derzeit die Frauen, die sich in den von ihr beleuchteten Berufsfeldern Anwältin, Notariat und Wirtschaftsprüferin erfolgreich betätigen, noch in der Unterzahl sind. In den Briefköpfen der großen Anwaltskanzleien stehen überwiegend Männer, diese bilden die Mehrzahl der Notare. Hierzu wies sie auf Projekte des DAV hin: ein Mentoring-Projekt, ein Projekt zur Vereinbarkeit „Anwältin und Mutter wie geht das?“, ein Projekt „Anwältinnen in die Aufsichtsräte" und den Anwältinnenpreis.

\section{Erste Schlussfolgerungen aus dem Kongress zum Zugang und zum Aufstieg im öffentlichen Dienst}

Der Kongress zeigte nicht nur, dass viel zu tun ist, sondern auch, wo der djb ansetzen kann. Es wurden auf dem Kongress Zusammenarbeitsangebote ausgesprochen, die aufzugreifen sein werden.

Mögliche Betätigungsfelder des djb, insbesondere im Bereich der Gesetzgebung (einschließlich Verordnungs- und Richtlinienrecht) sind:

1. Vergaberecht ${ }^{13}$

2. Beurteilungsrichtlinien und zugrunde liegende Laufbahnverordnungen $^{14}$
3. Frauenförderpläne mit begleitenden Personalführungs- und entwicklungsgesprächen müssen verpflichtend eingeführt werden. Vorgesetzte müssen Frauenförderung zu ihrer Aufgabe machen und dies muss Beförderungsvoraussetzung werden.

4. Gleichstellungsgesetze (Rechte und Stellung der Gleichstellungsbeauftragten, Gleichstellungspläne, Verbandsklagerecht, Individualklagerecht). Insbesondere folgende Schwachstellen in den bestehenden Gesetzen müssten beseitigt werden:

- Privatisierte Unternehmen fallen aus Anwendungsbereich des Bundesgleichstellungsgesetzes (BGleiG) und des Bundesgremienbesetzungsgesetzes (BGremG) heraus.

- Quote in $\int 8$ BGleiG gilt nicht für die Besetzung von Stellen, die durch Wahl erfolgt mit der Folge, dass die Besetzung von Bundesgerichten vollständig herausfällt.

- Bei den Gleichstellungsplänen nach dem BGleiG sind keine Nachbesserungspflichten und auch keine Sanktionen geregelt.

- Frühzeitige Beteiligung der Gleichstellungsbeauftragten funktioniert in der Praxis häufig nicht. Es besteht eine Diskrepanz zwischen recht umfangreichem Einspruchsrecht und der wesentlich eingeschränkteren Möglichkeit, Verstöße gerichtlich geltend zu machen.

- Hier müssen die Durchsetzungsmöglichkeiten verbessert und Sanktionen vorgesehen werden.

- Eine Verpflichtung zur Ausschreibung von Stellen ist gesetzlich vorzusehen.

- Die Steuerungswirkung der Gesetze ist insgesamt sehr mager. Regelungen, die zu einer besseren Steuerung führen würden, scheinen bewusst aus den Gesetzen heraus gelassen worden zu sein. So sind zum Beispiel altruistische Verbandsklagen nicht vorgesehen und Entgeltgleichheit ist nicht geregelt.

5. Eine externe Bewertung von Gleichstellungberichten wäre sinnvoll.

6. Jährliche Rankinglisten der Verwaltung (gegebenenfalls mit Negativ-Preisverleihung?) sowie eine Veröffentlichungspflicht von Berichten (Stichwort „public shaming ").

7. Daneben müssen Frauen besser lernen, Machtstrukturen zu durchschauen. Sie müssen Macht wollen und Seilschaften bilden und besser nutzen; insbesondere auch aktiv weibliche Nachwuchskräfte rekrutieren. Die Vereinbarkeit von Beruf und Familie schließlich muss endlich zum Männerthema gemacht werden.

11 Zu den Grenzen: vgl. BVerwG, Urteil v. 16.12.2010 - BVerwG 2 C 11.09-.

12 S. auch die noch immer hochaktuelle Stellungnahme des djb Nr. 05-O4 vom 15.2.2005, http://www.djb.de/Kom/K5/sn-178/, zugleich mit näheren Einzelheiten, u.a. Geschlechterquoten in den Beurteilungen, Evaluierung von Beurteilungen.

13 Vgl. Erster Gleichstellungsbericht, BT-Drs. 17/6240 S. 143 f., 157 f., 243.

14 Noch immer aktuell hierzu: Stellungnahme des djb v. 15.2.2005 (s. Fn. 12), zugleich mit näheren Einzelheiten, u.a. Geschlechterquoten in den Beurteilungen, Evaluierung von Beurteilungen und Veröffentlichung der Ergebnisse. 